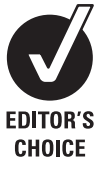

CHOICE

\title{
Prospective surveillance of drain associated meningitis/ventriculitis in a neurosurgery and neurological intensive care unit
}

\author{
S Scheithauer, ${ }^{1}$ U Bürgel, ${ }^{2}$ Y-M Ryang, ${ }^{2}$ G Haase, ${ }^{3}$ J Schiefer, ${ }^{4}$ S Koch, ${ }^{5}$ H Häfner, \\ S Lemmen ${ }^{1}$
}

\section{See Editorial Commentary,} p 1302

${ }^{1}$ Department of Infection Control and Infectious Diseases, University Hospital, RWTH Aachen, Germany; ${ }^{2}$ Department of Neurosurgery, University Hospital, RWTH Aachen, Germany; ${ }^{3}$ Institute of Medical Microbiology, University Hospital, RWTH Aachen, Germany; ${ }^{4}$ Department of Neurology, University Hospital, RWTH Aachen, Germany; ${ }^{5}$ Division of Infectious Diseases, Department of Internal Medicine, University Cologne, Germany

Correspondence to: Dr S Scheithauer, Department of Infection Control and Infectious Diseases, UK Aachen, RWTH Aachen, Pauwelsstrasse 30, 52074 Aachen, Germany; sscheithauer@ukaachen.de

Results were presented in part as an oral presentation at the 60th Annual Meeting of the DGHM, Dresden, Germany, 21-24 September 2008.

Received 14 October 2008 Revised 8 February 2009 Accepted 26 February 2009 Published Online First 16 March 2009

\section{ABSTRACT}

Objectives: There are currently no data available on drain associated infection occurrence related to the number of drainage days (DD), and thus drain associated infection rates. Therefore, a prospective surveillance study was conducted to determine drain associated infection rates and DD of hospital acquired external ventricular drain (EVD) and lumbar drain (LD) associated meningitis/ ventriculitis in a neurosurgery and a neurological intensive care unit.

Methods: All patients admitted in 2005 and 2006 were documented. Data on age, admitting diagnosis, type and duration of drain, duration of hospital stay and occurrence of meningitis were recorded and analysed statistically.

Results: A total of 1333 patients were included, amounting to $3023 \mathrm{DD}$. After exclusion of 15 contaminations, a total of 26 cases of meningitis were reported accounting for an overall device associated meningitis rate of 8.6 infections/1000 DD. Infections associated with LD seemed to occur more frequently (19.9/1000 DD) compared with EVD (6.3/1000 DD). The presence of intraventricular blood and previous trauma were significant risk factors for infection $(p=0.003 ; p=0.04)$. Finally, length of stay was significantly longer in meningitis patients $(p=0.0003)$. Coagulase negative staphylococci were the main pathogen $(56 \%)$ causing meningitis, followed by Staphylococcus aureus (25\%).

Conclusions: To the best of the authors' knowledge, this study represents the first to provide data on EVD as well as LD associated meningitis rates calculated per 1000 DD; a parameter that is well established for other invasive devices such as central venous and urinary tract catheters. However, further prospective studies are needed to investigate the possible risk factors for meningitis.

Nosocomial infection rates mainly correspond with the severity of illness and exposure to invasive devices. ${ }^{1}$ External ventricular drains (EVD) and lumbar drains (LD) are commonly used to control hydrocephalus and to monitor and decrease intracranial pressure. Ventriculostomy related infections are a serious problem in up to $40 \%$ of patients. $^{2-5}$ In contrast with shunt infections, infections derived from the use of devices are almost always classified as nosocomial due to the requirement for hospitalisation. ${ }^{67}$ To date, there have been studies on the incidence rate (IR) of meningitis, causative organisms and drain specific data in both neurosurgical and neurological settings. ${ }^{8-11}$ However, there have been no studies correlating meningitis with drain specific device days and, therefore, there are no data on drain associated incidence rates (DA-IR) relating meningitis to drainage days (DD).

The definition of drain associated meningitis is still not clearly defined and open to discussion. ${ }^{12}$ This may therefore lead to infection rates being overestimated when using the Centers for Disease Control (CDC) definition due to defining contaminations as infections.

The aim of this study was to test the suitability of the CDC definition for drainage associated meningitis and to identify the possible underlying risk factors for meningitis cases by determining utilisation rates (UR) and drain associated meningitis IR

\section{METHODS}

\section{Study wards and patients}

The study was performed prospectively from 1 January 2005 to 31 December 2006. The neurosurgical intensive care unit (NSICU) is a 14 bed ward and the neurological intensive care unit (NICU) is a seven bed ward; both care for adult patients only. To determine the total number of patients and patient days, only patients who stayed longer than $24 \mathrm{~h}$ were included. Each EVD and LD was counted separately to calculate the number of EVD as well as LD days, and the duration of drainage was documented for all patients in the NICU throughout the study and for patients in the NSICU for 2006.

LDs and EVDs were routinely inserted under sterile conditions at the patient's bedside, and seldom $(<5 \%)$ in the operating theatre. The Portex Epidural Catheter and Liquor Drainage Catheter Set (both Smiths Medical, Kirchseeon, Germany) were used for EVD and LD, respectively. Note that neither antibiotic nor silver impregnated catheters were used. A written protocol was followed for drainage placement, and the preferred insertion point of the EVD was the Kocher point on the right side with no tunnelling being carried out. No antibiotics were given prophylactically and no prophylactic catheter changes were performed. EVD and LD related infections were recorded only when the patients were admitted for longer than $48 \mathrm{~h}$. When infection was suspected, white cell count, protein and glucose levels for CSF and C reactive protein serum levels, total white blood cell counts as well as microbiologic examination were performed daily. Otherwise, tests were routinely carried out 3-4 times a week. CSF sampling was carried out by an aseptic technique following a 
written protocol by the neurosurgeon or the neurologist. Microbiological examination was performed using a conventional culture techniques as well as the BD Phoenix automated microbiology system (Becton Dickinson Diagnostic Systems, Heidelberg, Germany). Admitting diagnoses of patients needing drainage therapy were subarachnoid haemorrhage (SAH) $(55 \%)$, intracranial bleeding (ICB) (24\%), SAH+ICB (4\%), neoplasm $(6 \%)$, infection (3\%) and other (8\%). In 7.5\%, bleedings followed trauma. As no differences were found for the admitting diagnoses and surgical interventions for the NSICU and NICU patients, data were pooled for patients with meningitis.

\section{Surveillance and data collection}

Surveillance was performed by an experienced infection control team and instructed staff members using a nursing documentation sheet and midnight statistics. The following parameters were collected: sex, age, admitting diagnosis, surgical procedures, type of drainage, clinical signs indicative of meningitis/ventriculitis, parameters to determine inflammation from the serum and CSF, causative organism and frequency of isolation, resistance pattern and initiation of antibiotics for meningitis/ventriculitis. Firstly, each detection of a pathogen in the CSF and/or at the tip of the drain was documented as meningitis/ventriculitis according to the CDC definition. Moreover, the combination of at least one typical clinical sign indicating meningitis (eg, neck stiffness), at least one pathological parameter in the CSF (elevated cell count, decreased glucose level in our cases) and the initiation of antibiotics due to suspicion of meningitis lead to the diagnosis of meningitis as defined by the CDC (second criterion of the case definition). Secondly, contaminations were excluded after investigation of these cases suspicious for contamination only according to the criteria derived from our results. Finally, meningitis cases were excluded if meningitis followed a systemic infection (eg, central venous line sepsis).

For correlation of meningitis with DD, admitting diagnosis and age data were available for all NICU patients cared for with drainage and for all NSICU patients with drainage admitted during 2006. Student's t test (level of significance $p<0.05 ; 95 \%$ confidence interval) and $\chi^{2}$ test were used to calculate means, differences and odds ratios.

\section{RESULTS \\ Microbiology}

In 40 nosocomial drain associated cases of meningitis, a pathogen was isolated. In four patients, more than one episode of ventriculitis with different pathogens was detected. In general, coagulase negative staphylococci (CoNS) was found to be the main pathogen, followed by Staphylococcus aureus (SA). Bloodstream infection was found to occur in only one case after meningitis (due to SA). Interestingly, in four EVD/LD associated infections, methicillin resistant SA (MRSA 10\%) was isolated, accounting for an MRSA rate of $57 \%$ (4/7) for all cases of SA induced meningitis. Finally, two-thirds of CoNS were found to be oxacillin resistant whereas none of the other pathogens showed any unusual antibiotic resistance pattern. Data on species, frequency of the isolated organism, relation to the device and fulfilling the CDC case definition are provided in table 1 .

\section{Definition of meningitis}

Patients diagnosed with meningitis from CSF analysis did not often comply with the second criterion of the CDC definition, especially when it was CoNS induced. For example, in only 13 of 41 patients (32\%) could clinical signs be described. A decreased glucose level in CSF was detected in 15 of the 41 $(37 \%)$ meningitis patients and was found in all cases of meningitis due to SA, E faecalis, Gram negative rods and yeasts. In contrast, in $90 \%$ (37/41) of our patients, white blood cell counts were elevated in the CSF and ranged from 16 to 4270, with an average of $868 \mathrm{cells} / \mathrm{ml}$ CSF specimen. Of note was that more than $90 \%$ of the analysed CSF specimens were at least moderately bloody (++) and more than $50 \%$ were highly blood stained (++++). In 22 of 41 cases (54\%), patients were prescribed antibiotics against possible meningitis.

To ascertain whether the frequency of isolating CoNS allows us to distinguish true infections from contaminations we subdivided patients with regard to the number of positive CSF results.

Only cases in which a positive CSF specimen was followed by at least one negative one were documented as "isolation once". We found that isolating CoNS twice or more seemed to be indicative of meningitis infection whereas isolating CoNS in only one sample seemed to be indicative of contamination only (see table 2).

\section{Device utilisation and nosocomial infection rates}

During the 2 year prospective surveillance study, a total of 1333 (NSICU $\mathrm{n}=746$; NICU $\mathrm{n}=587$ ) patients were enrolled, accounting for a total of 11822 (NSICU $n=7046$; NICU $\mathrm{n}=4776$ ) patient days (PD). A total of 3023 DD were observed of which 503 were LD days (LD-D) and 2520 EVD days (ED-D). The majority of catheters were used on the NSICU with 2732 DD, accounting for 2267 ED-D and 465 LD-D. On the NICU, a total of 291 DD were counted of which 253 were ED-D and 28 LD-D. Thirty-seven of 1333 patients accounted for 41 episodes of nosocomial device associated meningitis/ventriculitis with 37 and four infections occurring in the NSICU and NICU, respectively. Results for the device utilisation rate, IR of meningitis/ventriculitis (calculated per 100 patients $=100$ patients), incidence densities and device associated meningitis/ ventriculitis rates (DA-IR, calculated per DD) as well as the formulae used to derive the results are provided in table 3 . Exclusion of the cases with contaminants only resulted in considerably lower overall DA-IR (8.6 vs 13.6 infections per 1000 DD; EVD 6.3 vs 8.7 infections per 1000 DD; LD 19.9 vs 37.8 infections per 1000 DD).

\section{Characterisation of meningitis and meningitis patients}

Mean age of patients having had meningitis was 49.09 years (SD 12.75; range 26-77) and no difference from drainage patients without infection was found (53.47 years, SD 15.35; range $15-85 ; p=0.20$ ). Mean length of hospital stay was 34.70 (SD 19.18; range 4-113) days for patients with drain and without meningitis compared with 51.46 (SD 26.64; range 7114) days for meningitis patients. The differences between patients without and with infection were statistically significant $(p=0.0003)$ and for EVD were 34.48 days (SD 20.82) without infection versus 55.56 days (SD 25.38) with infection $(p=0.0006)$. Mortality in the meningitis group $(3 / 26$, in one case due to infection) did not differ significantly from mortality in patients with drains and without meningitis (odds ratio 1.34, $95 \%$ confidence interval 0.35 to $5.05 ; \mathrm{p}=0.67$ ).

Meningitis occurred more often in patients with LD than EVD (table 1); however, the difference was not statistically significant (table 4). Association of admitting diagnosis with infection was seen only for trauma previous to bleeding. 
Table 1 Causative organisms and diagnosis of infection according to the Centers for Disease Control definition

\begin{tabular}{lccccc}
\hline Causative organism & EVD & LD & $\boldsymbol{\Sigma}(\%)$ & $\begin{array}{l}\text { Criterion } \mathbf{1} \\
\text { only }(\%)\end{array}$ & $\begin{array}{c}\text { Criterion } \\
\mathbf{1 + 2}(\%)\end{array}$ \\
\hline$\Sigma$ (CoNS) & 13 & 16 & $29(73)$ & $25(86)$ & $4(14)$ \\
$\Sigma$ (S aureus), divided into & 3 & 4 & $7(18)$ & $3(43)$ & $4(57)$ \\
$\quad S$ aureus, MSSA & 2 & 1 & $3(8)$ & $2(67)$ & $1(33)$ \\
$\quad$ S aureus, MRSA & 1 & 3 & $4(10)$ & $1(25)$ & $3(75)$ \\
Enterococcus faecalis & 1 & 0 & $1(2.5)$ & $0(0)$ & $1(100)$ \\
Acinetobacter baumannii & 1 & 0 & $1(2.5)$ & $0(0)$ & $1(100)$ \\
Pseudomonas aeruginosa & 1 & 0 & $1(2.5)$ & $0(0)$ & $1(100)$ \\
Candida albicans & 1 & 0 & $1(2.5)$ & $0(0)$ & $1(100)$ \\
$\Sigma$ (all microorganism) & 20 & 20 & 40 & $28(70)$ & $12(30)$ \\
\hline
\end{tabular}

Criterion 1, detection of a microorganism in CSF.

Criterion 2, at least one clinical symptom and at least one positive laboratory result and starting of antimicrobial therapy.

CoNS, coagulase negative staphylococci; EVD, external ventricular drain; LD, lumbar drain; MRSA, methicillin resistant Staphylococcus aureus; MSSA, methicillin sensitive Staphylococcus aureus.

Detection of intraventricular blood by cranial CT was significantly correlated with meningitis (table 4).

Mean duration of drainage placement was 11.11 days (SD 7.33; range 1-35) for EVD in the non-infected patients and 12.56 days (SD 9.02; range 1-37) in meningitis patients with no significant difference $(p=0.48)$. Mean duration of LD placement in the non-infected patients was 6.92 days (SD 4.91, range $1-22$ ) in contrast with 8.20 days (SD 5.98; range 3-23) in the meningitis group with no significant difference $(p=0.49)$. However, the longer EVD placement differed significantly from the LD duration $(p=0.0003)$.

Drainage duration of more than 9 days did not correlate with meningitis infection in our group. For correlation of the occurrence of meningitis and the drainage day-before and after excluding contaminations-see fig 1 .

\section{DISCUSSION}

The literature on surveillance data about nosocomial infections, knowledge about the pathophysiology as well as risk factors is steadily increasing. ${ }^{13}{ }^{14}$ However, despite there being many studies on, and subsequently guidelines about, preventing infections, especially those that are device associated, drain related infection occurrence and number of $\mathrm{DD}$, and thus drain related infection rates, are lacking, ${ }^{15-17}$ thereby severely hampering meaningful comparison and benchmarking, and the development of effective disease prevention guidelines/measures. Intraventricular haemorrhage was previously identified as a risk factor for meningitis, with a reported IR of up to 37.5/100P..$^{2} 18$ Therefore, patients in this study have to be classified as a high risk group and intraventricular haemorrhage was also identified as correlating with meningitis. In our study, we obtained a mean device associated incidence rate (DA-IR) of 8.6 infections per $1000 \mathrm{DD}$ after eliminating contaminants, which to our knowledge represent the first reported DA-IRs calculated per DD. DA-IRs per DD can be used as surveillance parameters and are well established for other device associated infections (eg, central line associated bloodstream infection) and are accepted as representing the most stable and valid parameters for these device associated infections. ${ }^{19}$

However, there are difficulties in comparing published data, especially the surveillance parameter. For example, some investigators document overall, not only device associated, infections, and in some studies data on meningitis and ventriculitis are presented separately, ${ }^{8}{ }^{10}$ while others are divided
Table 2 Frequency of isolating CoNS in relation to diagnostic criteria

\begin{tabular}{|c|c|c|}
\hline CoNS & $\begin{array}{l}\text { Isolation once } \\
(n=15)(\%)\end{array}$ & $\begin{array}{l}\text { Isolation twice or more } \\
(\mathrm{n}=10)(\%)\end{array}$ \\
\hline \multicolumn{3}{|l|}{ CDC definition } \\
\hline Criterion 1 only & $15(100)$ & $6(60)$ \\
\hline Criterion $1+2$ & $0(0)$ & $4(40)$ \\
\hline LD & $9(60)$ & $4(40)$ \\
\hline EVD & $6(40)$ & $6(60)$ \\
\hline Symptomatic + & $0(0)$ & $4(40)$ \\
\hline WBC CSF (M/ Ml) & 341 & 1064 \\
\hline Glucose level CSF (mg/dl) & 65 & 64 \\
\hline Protein CSF (mg/dl) & 0.8 & 0.7 \\
\hline Antibiotic therapy + & $3(20)$ & $7(70)$ \\
\hline
\end{tabular}

CDC, Centers for Disease Control; CoNS, coagulase negative staphylococci; EVD, external ventricular drain; LD, lumbar drain; WBC, white blood cell count.

into LD and EVD associated meningitis. For example, Schade and colleagues ${ }^{15} 16$ calculated an IR of 7/100 patients with LD and 15/100 patients with EVD and Orsi et al obtained an IR of 3/100 patients with $\mathrm{LD}$ or EVD. ${ }^{17} \mathrm{~A}$ basic difference is due to comparison of infections in 100 patients by some $e^{810}$ and in 100 patients with drainage by others. ${ }^{15-17}$ Moreover, mainly IR and incidence densities (calculation per 100 patients or per patient days) are given, but DA-IR calculated per DD (DA-IR) are not. ${ }^{4} 581012$ 20-25 Calculating the IR per 100 patients in our group with an IR of 3.08/100 patients (0.7 in the NICU and 5.0 in the NSICU) fell within the broad range of published data on meningitis associated with shunt or drain..$^{11}{ }^{12}{ }^{15-17}$ 20-28 In other studies where the $\mathrm{DD}$ were taken into account, infections were related to the number of device kits or procedures, but not to DD. ${ }^{11}{ }^{22}$ For example, Coplin et al calculated the LD associated IR to be $4.2 \%$ related to kits. ${ }^{22}$ In comparison with our results, Dettenkofer et al reported slightly lower IRs in the NICU (1.2/ $100 \mathrm{P})$ than in the NSICU $(1.8 / 100 \mathrm{P}) .{ }^{8}{ }^{10}$ However, this apparent discrepancy in our study population was not confirmed when comparing DA-IR (NICU 10.3; NSICU 8.4 infections per $1000 \mathrm{DD}$ ). Thus the difference seems only to be simulated because of the varying UR for both ICUs. In contrast, the risk of infection seems to be higher for LD with a DA-IR of 19.9 infections per $1000 \mathrm{DD}$ than for EVD with a DA-IR of 6.3 infections per $1000 \mathrm{DD}$ in our study. There is no obvious explanation for this finding and thus further studies are needed.

To summarise, DA-IRs calculated per DD are accepted as representing the most stable and valid parameters that can be used as a benchmark to help develop effective preventative measures, thereby lowering the level of infection and improving

Table 3 Formulae for calculation and derived results

\begin{tabular}{lcllc}
\hline & $\begin{array}{l}\text { DUR: DD } \\
\times \mathbf{1 0 0} / \mathbf{P D}\end{array}$ & $\begin{array}{l}\text { IR: DAI/ } \\
\mathbf{1 0 0} \mathbf{P}\end{array}$ & $\begin{array}{l}\text { ID: DAI } \\
\times \mathbf{1 0 0 0} / \mathbf{P D}\end{array}$ & $\begin{array}{l}\text { DA-IR: DAI } \\
\times \mathbf{1 0 0 0} / \mathbf{D D}\end{array}$ \\
\hline LD(NSICU) & 6.6 & 1.3 & 1.4 & 21.5 \\
EVD(NSICU) & 32.2 & 1.7 & 1.8 & 5.7 \\
$\Sigma$ (NSICU) & 38.8 & 3.1 & 3.3 & 8.4 \\
LD(NICU) & 0.8 & 0.0 & 0.0 & 0.0 \\
EVD(NICU) & 5.3 & 0.5 & 0.6 & 11.9 \\
$\Sigma$ (NICU) & 6.1 & 0.5 & 0.6 & 10.3 \\
$\Sigma$ (LD) & 4.3 & 0.8 & 0.8 & 19.9 \\
$\Sigma$ (EVD) & 21.3 & 1.2 & 1.1 & 6.3 \\
$\Sigma$ & 25.6 & 2.0 & 2.2 & 8.6
\end{tabular}

DAl, device associated infections; DA-IR, device associated infection rate (calculated per device days); DD, device days; DUR, device utilisation rate; EVD, external ventricular drain; ID, incidence density; IR, incidence rate; LD, lumbar drain; NICU, neurological intensive care unit; NSICU, neurosurgical intensive care unit; $\mathrm{P}$, patient; PD, patient days. 
Table 4 Risk factors analysis for meningitis

\begin{tabular}{lll}
\hline Meningitis & OR (Cl 95\%) & p $(<\mathbf{0 . 0 5})$ \\
\hline LD versus EVD & $1.39(0.58-3.30)$ & 0.46 \\
Previous trauma & $3.21(1.00-10.33)$ & 0.04 \\
Intraventricular blood (cranial CT; NSICU) & $4.93(1.57-15.45)$ & 0.003 \\
LD placement $>9$ days & $1.38(0.29-6.48)$ & 0.68 \\
EVD placement $>9$ days & $0.61(0.25-1.48)$ & 0.27
\end{tabular}

EVD, external ventricular drain; LD, lumbar drain; NSICU, neurosurgical intensive care unit.

patient outcome. Finding CoNS to be the most frequent pathogen of drain associated meningitis (56\%) was not surprising. ${ }^{29}$ Data published on shunt infections showed staphylococcal species to be the cause of at least $60 \%$ of infections and mainly due to CoNS, followed by Gram negative rods, found in up to $20 \%$ of cases. ${ }^{8} 122022253031$ It is standard practice to follow the CDC definitions for which every detected microorganism has to be evaluated as a potential causative organism. ${ }^{2} 101729$ However, some investigators only included bacteriologically proven infections, ${ }^{22}{ }^{24}$ and others excluded cases when CoNS and other skin commensals such as Corynebacterium $\mathrm{sp}$ were isolated using the investigators' own definitions and in these case they were classified as contaminants or colonisation of the catheter only ${ }^{16}{ }^{32}$ However, care must be taken not to oversimplify possible causative organisms such as CoNS and other factors (eg, frequency of isolation should also be taken into consideration to help make a more accurate diagnosis in the cases of CoNS). ${ }^{5} 8121620-25$ Of note is that studies on shunt infections have already shown that the absence of fever and indicators of meningitis does not rule out the possibility of meningitis infection. ${ }^{17}{ }^{33}$ This finding is partly to be expected when one considers the low virulence of CoNS compared with, for example, $S$ aureus. A non-specific and unsuspicious presentation of drain associated meningitis makes a rapid and appropriate diagnosis difficult. Moreover, pathological CSF parameters also have to be interpreted with caution as patients with shunts or drainages often show abnormal CSF profiles regardless of infection. ${ }^{173}$ There is also no clear cut-off value on how to interpret elevated white cell counts by different degrees of blood stained CSF although patients with SAH or ICB have been reported to show elevated cell counts of more than 100/ $\mathrm{mm}^{3}$ in the case of bacteriologically proven meningitis. ${ }^{22}{ }^{34} \mathrm{In}$ addition, whether the detection of CoNS or other skin commensals in one single CSF specimen is sufficient to diagnose drain associated meningitis is questionable. ${ }^{20-22}$ As known from the blood culture diagnosis, the frequency of isolating CoNS is a well established additional parameter to help between discriminate contamination and infection. ${ }^{35}$ Therefore, we investigated whether the frequency of CSF samples with CoNS in our setting is of additional value in discriminating true meningitis from contaminations only. None of our patients in whom CoNS was detected only once showed clinical signs of meningitis. The misinterpretation of CoNS contaminants as indicative of true infections has implications for both patient care and hospital quality assurance. Excluding the cases diagnosed by isolating CoNS only once resulted in considerable lower device associated infection rates ( 8.6 vs 13.6 infections per $1000 \mathrm{DD})$ in our patient group. The modified case definition since January 2008 (National Reference Laboratory for the Surveillance of Nosocomial Infections, Berlin, Germany) takes into consideration the possibility of misinterpretation of contaminants. ${ }^{36}$ Therefore, we would like to suggest the number of CSF samples with isolation of CoNS as an additional criterion in discriminating true meningitis infections from contaminations. We found that trauma bleeding after head injury and the presence of intraventricular blood were significant risk factors for meningitis. In the presented study, we found no obvious relationship between infection and $\mathrm{DD}$. Meningitis patients showed a significantly longer duration of hospital stay than drainage patients without meningitis. In addition to our risk factor analysis, the techniques of drainage placement (eg, tunnelling), the influence of prophylactically given antibiotics and the impact of the place where the insertion procedure was carried out are of interest and should be evaluated in further studies.

In conclusion, to the best of our knowledge, we present the first IRs calculated with regard to DD for both EVD and LD associated meningitis/ventriculitis in a NSICU and NICU. We would like to encourage the implementation of a routine surveillance programme for both EVD and LD associated meningitis/ventriculitis in hospitals caring for those patients. A significant reduction of device associated infections such as central line associated bloodstream infection could be proven by generating these data. ${ }^{37}$

Our findings show that risk factors for meningitis were the presence of intraventricular blood and previous trauma. In addition, length of hospital stay was significantly longer in
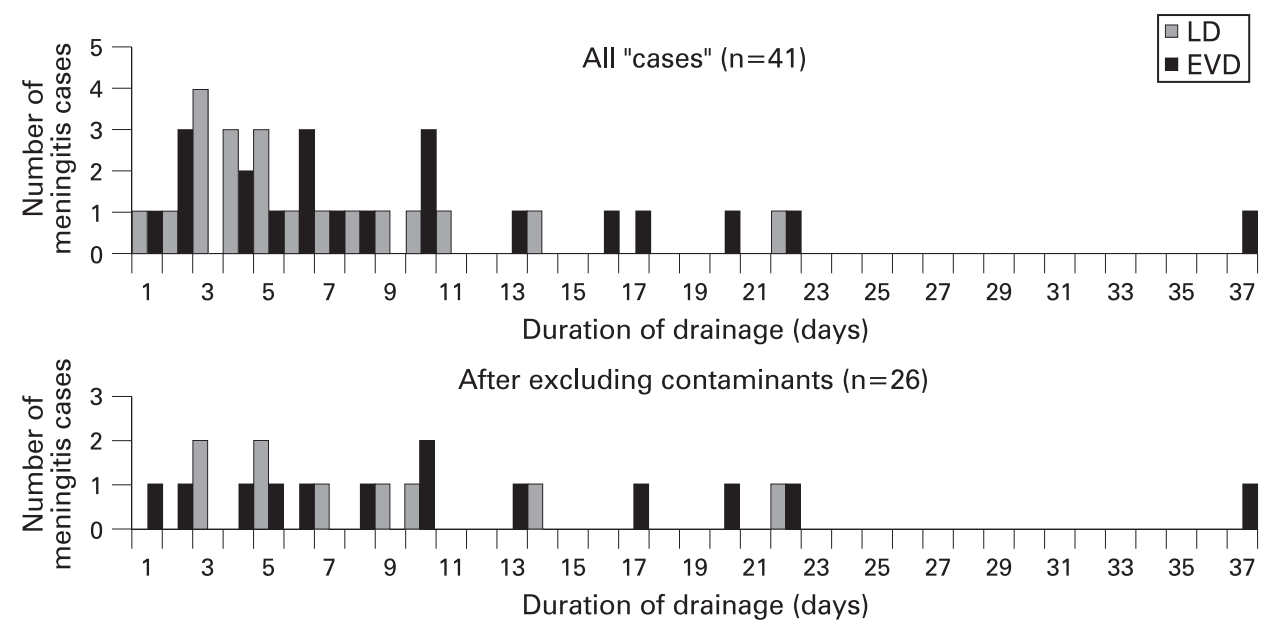

Figure 1 Occurrence of meningitis related to drainage day. EVD, external ventricular drain; LD, lumbar drain. 
patients with meningitis. However, the commonly used definition of drain associated meningitis/ventriculitis (by the $\mathrm{CDC}^{38}$ ) may be limited in excluding true contaminants, thus resulting in an over reporting of infections, especially overestimating the role of CoNS. Therefore, we would like to suggest that the number of isolated CoNS be taken into account as an additional criterion to rule out contaminants. Finally, further investigations are warranted in order to provide a more effective framework for better diagnosing and analysing risk factors of infection to improve patient outcome.

\section{Competing interests: None.}

Ethics approval: Ethics approval was decided by request; no formal proposal was required as no intervention was planned, only a description of anonymised data. Professor Dr Schmalzing, Director of the Ethics Committee, University of Aachen.

Provenance and peer review: Not commissioned; externally peer reviewed.

\section{REFERENCES}

1. Daschner FD, Frey P, Wolff G, et al. Nosocomial infections in intensive care wards: a multicenter study. Intensive Care Med 1982;8:5-9

2. Stenager $\mathbf{E}$, Gerner-Smidt $\mathrm{P}$, Kock-Jensen $\mathrm{C}$. Ventriculostomy-related infections - an Epidemiological Study. Acta Neurochir 1986;83:20-3.

3. Mayhall CG, Archer NH, Lamb VB, et al. Ventriculostomy-related infections. N Engl J Med 1984;310:553-9.

4. Holloway K, Barnes T, Choi S, et al. Ventriculostomy infections: the effect of monitoring duration and catheter exchange in 584 patients. J Neurosurg 1996:85:419-24.

5. Popp W, Müller 0, Schoch B, et al. Infektionsraten bei externen Ventrikeldrainagen. Hyg Med 2004;29(Suppl1).

6. Buckwold FJ, Hand R, Hansebout RR. Hospital-acquired bacterial meningitis in neurosurgical patients. J Neurosurg 1977;46:494-500.

7. Durand ML, Calderwood SB, Weber DJ. Acute bacterial meningitis in adults. A review of 493 episodes. N Engl J Med 1993;328:21-8.

8. Dettenkofer M, Ebner W, Els Th, et al. Surveillance of nosocomial infections in a neurology intensive care unit. J Neurol 2001;248:959-64.

9. Shafer SQ, Brust JC, Healton EB, et al. Hospital-acquired morbidity on a neurology service. J Natl Med Assoc 1993;85:31-5.

10. Dettenkofer M, Ebner W, Hans FJ, et al. Nosocomial infections in a neurosurgery intensive care unit. Acta Neurochir 1999;141:1303-8.

11. Korinek AM, Reina M, Boch $\mathrm{AL}$, et al. Prevention of external ventricular drainrelated ventriculitis. Acta Neurochir 2005;147:39-46.

12. Lozier AP, Sciacca RR, Romagnoli MF, et al. Ventriculostomy-related infections-a critical review. Neurosurgery 2002:51:170-81.

13. Gaynes RP, Horan TC. Surveillance of nosocomial infections. In: Marshall CG, ed. Hospital epidemiology and infection control. Baltimore: Williams and Willkins, 1999:1018-31.

14. Haley RW, Culver DH, White JW, et al. The efficacy of infection surveillance and control programs in preventing nosocomial infections in US hospitals. Am J Epidemiol 1985;212:182-205.

15. Schade RP, Schinkel J, Roelandse FW, et al. Lack of value of routine analysis of cerebrospinal fluid for prediction and diagnosis of external drainage-related bacterial meningitis. J Neurosurg 2006;104:101-8.
16. Schade RP, Schinkel J, Visser LG, et al. Bacterial meningitis caused by the use of ventricular or lumbar cerebrospinal fluid catheters. J Neurosurg 2005;102:229-34.

17. Orsi GB, Scorzolini L, Franchi $C$, et al. Hospital-acquired infection surveillance in a neurosurgical intensive care unit. J Hosp Infect 2006;64:23-9.

18. Noetzel MG, Baker RP. Shunt fluid examination: risks and benefits in the evaluation of shunt malfunction and infection. J Neurosurg 1984;61:328-32.

19. Edwards JR, Peterson KD, Andrus ML, et al, NHSN Facilities. National Healthcare Safety Network (NHSN) Report, data summary for 2006, issued June 2007 Am J Infect Control 2007:35:290-301.

20. Pfisterer W, Mühlbauer $M$, Czech $T$, et al. Early diagnosis of external ventricular drainage infection: results of a prospective study. J Neurol Neurosurg Psychiatry 2003;74:929-32.

21. Berger C, Schwarz S, Schaebitz WR, et al. Serum procalcitonin in cerebral ventriculitis. Crit Care Med 2002;30:1778-81.

22. Coplin WM, Avellino AM, Kim DK, et al. Bacterial meningitis associated with lumbar drains: a retrospective cohort study. J Neurol Neurosurg Psychiatry 1999;67:468-73.

23. Pfausler B, Beer R, Engelhardt $K$, et al. Cell index: A new parameter for the early diagnosis of ventriculostomy (external ventricular drainage)-related ventriculitis in patients with intraventricular hemorrhage? Acta Neurochir 2004;146:477-81.

24. Park P, Garton HJ, Kocan MJ, et al. Risk of infection with prolonged ventricular catheterization. Neurosurgery 2004;55:594-9.

25. Wong GK, Poon WS, Wai S, et al. Failure of regular external drain exchange to reduce cerebrospinal fluid infection: result of a randomised controlled trial. J Neurol Neurosurg Psychiatry 2002;73:759-61.

26. Bayston R. Infections associated with central nervous system implants. In: Seifert H, Jansen B, Farr BM, eds. Catheter-related infections. London: Marcel-Dekker, 1997:325.

27. Lam CH, Villemure JG. Comparison between ventriculoatrial and ventriculoperitonea shunting in the adult population. Br J Neurosurg 1997;11:43-8.

28. Hopmanns TEM, Blok HEM, Troelstra A, et al. Prevalence of hospital-aquired infections during successive surveillance surveys conducted at a university hospital in the Netherlands. Infect Control Hosp Epidemiol 2007;28:459-65.

29. Huang CR, Lu CH, Wu JJ, et al. Coagulase-negative staphylococcal meningitis in adults: clinical characteristics and therapeutic outcomes. Infection 2005;33:56-60.

30. Morris A, Low DE. Nosocomial bacterial meningitis, including central nervous system shunt infections. Infect Dis Clin North Am 1999;13:735-50.

31. De Bels D, Korinek AM, Bismuth R, et al. Empirical treatment of adult postsurgical nosocomial meningitis. Acta Neurochir 2002;144:989-95.

32. Hader WJ, Steinbok PM. The value of routine cultures of the cerebrospinal fluid in patients with external ventricular drains. Neurosurgery 2000:46:1149-55.

33. Kaufman BA, Tunkel AR, Pryor JC, et al. Meningitis in the neurosurgical patients. Infect Dis Clin North Am 1990;4:677-701.

34. Conen A, Walti LN, Merlo A, et al. Characteristics and treatment outcome of cerebrospinal fluid shunt-associated infections in adults: a retrospective analysis over an 11-year period. CID 2008;47:73-82.

35. Tokars Jl. Predictive value of blood cultures positive for coagulase-negative staphylococci: implications for patient care and health care quality assurance. CID 2004:39:333-41.

36. Nationales Referenzzentrum (NRZ) für Surveillance von nosokomialen Infektionen. Definition nosokomialer Infektionen (CDC-Definitionen). Berlin 2008, 6 Auflage, 19-20.

37. Gastmeier P, Sohr D, Schwab F. Ten years of KISS: The most important requirements for Success. J Hosp Infect 2008;70:11-16.

38. Horan TC, Andrus M, Dudeck MA. CDC/NHSN surveillance definition of health careassociated infection and criteria for specific types of infections in the acute care setting. Am J Infect Control 2008;36:309-32. 